\title{
Coherent nuclear wave packet dynamics of laurdan launched by intramolecular charge transfer
}

\author{
S. Y. Kim and T. Joo \\ Department of Chemistry, Pohang University of Science and Technology (POSTECH), Pohang 790- \\ 784, South Korea
}

\begin{abstract}
Coherent nuclear wave packets in the product state launched by the ultrafast intramolecular charge transfer are observed by time-resolved fluorescence with 40 fs time resolution. Direct information on reaction coordinates and structural changes can be obtained.
\end{abstract}

\section{Introduction}

Intramolecular charge transfer (ICT) is one of the most important reactions for its usefulness in applications such as the bio-imaging as well as the fundamental importance. As shown in Figure 1, laurdan (6-dodecanoyl-2-dimethylaminonaphtalene) exhibits dual fluorescence from the locally excited (LE) and ICT states, where the ratio depends sensitively on the solvent polarity. This feature has been exploited to probe the local polarity by generalized polarization images [1,2]. Laurdan, an analogue of DMABN (4-dimethylaminobenzonitrile), which is the benchmark system of ICT reaction, consists of an electron-donating dimethylamino group and an electron-withdrawing carbonyl group on the opposite side of the naphthalene. The ICT reaction may proceed through the twisting of the dimethylamino group, although its dynamics including the structural change still remains under intense investigation because of the conflicting experimental and theoretical reports $[3,4]$.

Coherent nuclear wave packets in the excited states can be launched by an ultrafast chemical reaction faster than the vibrational period just as the Franck-Condon transition. The wave packets generated by the ultrafast ICT evolve in time on the ICT surface, and their oscillations include normal modes with large vibrational displacements between the ground and ICT states. Therefore, direct observation of the coherent nuclear wave packet dynamics on the product surface may provide valuable information on the reaction coordinates and the molecular structures of the involved states [5]. In this work, we have studied the ultrafast ICT dynamics by time-resolved fluorescence (TRF), which gives the signal from the excited state exclusively. TRF of laurdan in methanol was measured by fluorescence up-conversion via two-photon excitation with $40 \mathrm{fs}$ time resolution, which was high enough to resolve the wave packet oscillations up to $700 \mathrm{~cm}^{-1}$ [6].

\section{Results and discussion}

To study the molecular dynamics of the ICT reaction, we have measured the TRF of laurdan in methanol at $520 \mathrm{~nm}$. Note that the detection wavelength indicated in Figure 1 is distant enough from the LE band to consider the emission arising solely from the ICT state. The TRF signal was fitted to 
a sum of exponentials to give three rise components of $<60 \mathrm{fs}$ (ultrafast ICT), 3 ps and $20 \mathrm{ps}$. In addition, the oscillation of the TRF signal, which is obvious as in Figure 2, was analyzed by the linear prediction singular value decomposition (LPSVD) method and the Fourier transform. It is remarkable in that only one prominent mode at $496 \mathrm{~cm}^{-1}$ was observed without any other lowfrequency vibrations. This represents the first observation of the coherent wave packet motion in the product ICT state by TRF.

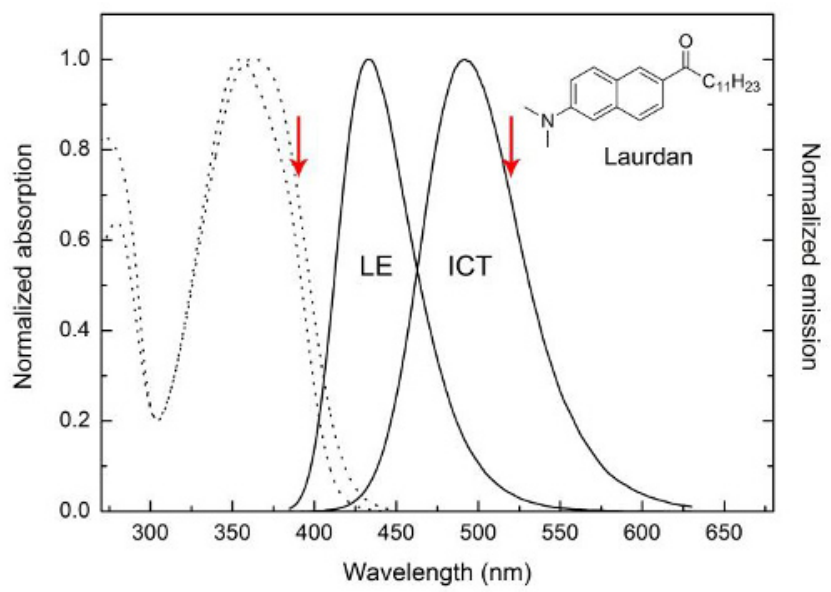

Fig. 1. Molecular structure of laurdan and its steady-state spectra. The dotted lines show the absorption spectra and the solid lines show the emission spectra in chloroform and methanol, respectively. Arrows indicate the excitation wavelength (two-photon excitation of $780 \mathrm{~nm}$ ) and the detection wavelength $(520 \mathrm{~nm})$ in the TRF measurement.

In order to identify the vibrational mode observed in the TRF, quantum mechanical calculation was performed for laurdan with the dodecyl group replaced by ethyl. Geometry optimizations and frequency calculations were carried out at the B3LYP/6-31G ${ }^{*}$ level for the ground state and CIS/6$31 \mathrm{G}^{*}$ level for the first and second excited states by using the Gaussian 03 package. The first excited state, called ICT(P), has a planar structure parallel to the naphthalene backbone, while the second excited state, called ICT(T), has a twisted dimethylamino group with a dihedral angle of $64.3^{\circ}$. Similar to the Franck-Condon transition, when the reaction occurs instantaneously, vibrational modes with large displacements between the ground and product ICT (not LE) states will be excited preferentially. That is, we can define a general Huang-Rhys factor coupled to the reaction (electronic transition) beyond the Franck-Condon transition. Hence, we can estimate which vibrational modes comprise the wave packet in the ICT state by calculating the projection of the displacement between reactant (ground state) and product (ICT state) onto the normal modes of the product state.

We have calculated the normal-mode-projected displacements for two cases; ground $\rightarrow \mathrm{ICT}(\mathrm{P})$ and ground $\rightarrow \mathrm{ICT}(\mathrm{T})$. Although the match is not perfect, the first case agrees with the experiment much better than the second. In the calculation from ground state onto the ICT(P), there are 5 normal modes with large displacements and all of them are in-plane. Among them, modes 9, 14, and 18 are similar in the rocking motion of the entire molecule including donor and acceptor. On the other hand, the conformational changes in modes 20 and 21 (Figure 2 (c)) are mainly concentrated on the ring stretching motion. Modes $20\left(483 \mathrm{~cm}^{-1}\right)$ and $21\left(498 \mathrm{~cm}^{-1}\right)$, which have the largest reorganization energies, can be assigned to the experimentally observed $496 \mathrm{~cm}^{-1}$ mode. Note that only the $496 \mathrm{~cm}^{-1}$ mode was observed even though the calculated results contain some low-frequency modes with large displacements. The lower frequency modes, involving the movement of the donor and the acceptor, have been expected to induce the ICT reaction, but they are absent in the experiment. From this, we can draw a conclusion that is in strong contrast with the current theory; the ring stretching motion in the aromatic (naphthalene) backbone is much more important in the ICT dynamics of laurdan than the twisting or planarization of the dimethylamino group. 

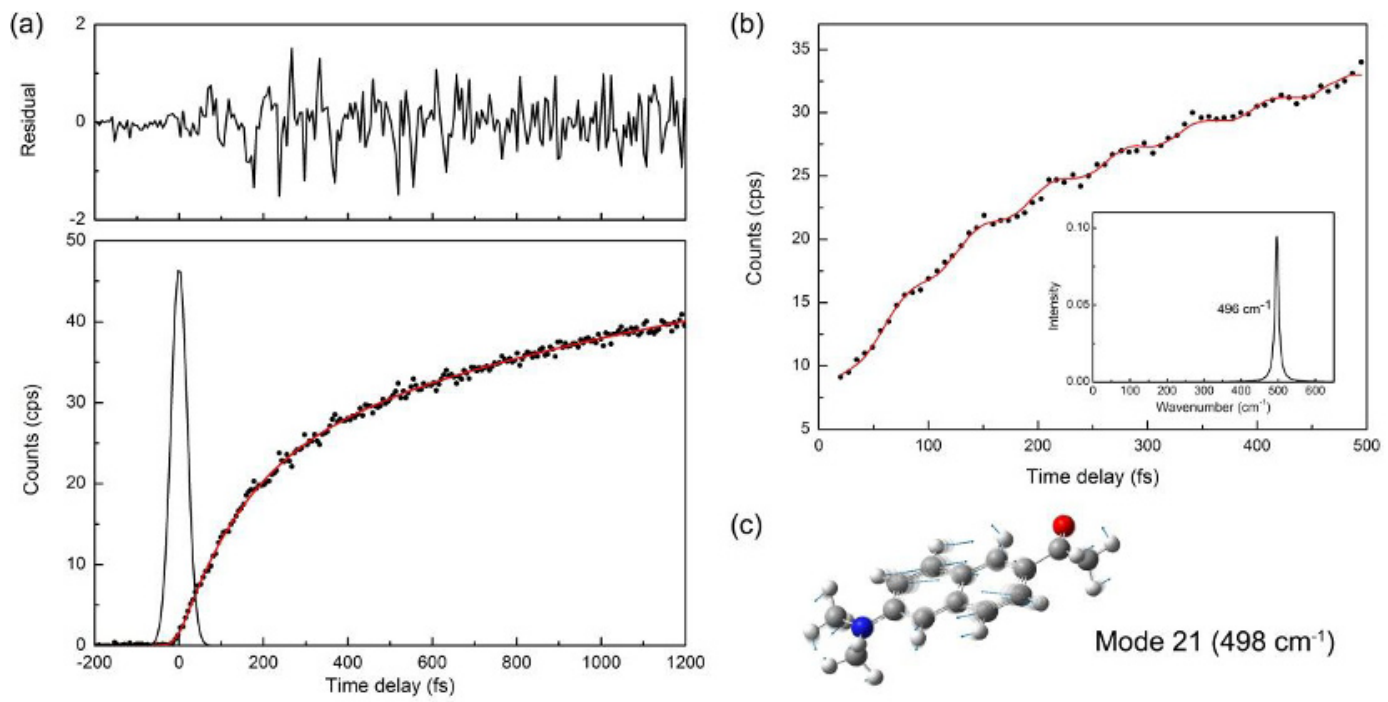

(c)

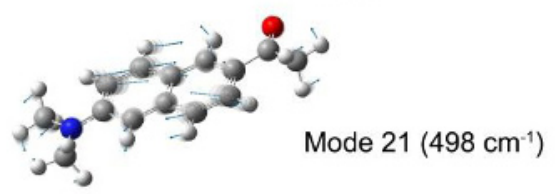

Fig. 2. (a) TRF of laurdan in methanol at $520 \mathrm{~nm}$. Red solid line is the best fit to three exponentials and a Gaussian peak at time zero is the instrument response. The residual is a difference between the TRF signal and the fitted line, and it shows a clear oscillation. (b) Frequency spectra of the oscillation components obtained from the LPSVD method. (c) The $21^{\text {st }}$ normal mode of the ICT(P) state. It corresponds to the ring stretching motion.

\section{Conclusions}

Coherent nuclear wave packet motion from the product ICT state is clearly observed. This was possible by the ultimate time resolution in the TRF measurement. In comparison with the calculated results, the observed oscillation can be assigned to the stretching motion of the naphthalene ring, not the dimethylamino group. This provides a new insight on the ICT dynamics.

\section{References}

1. T. Parasassi, E. K. Krasnowska, L. Bagatolli, E. Gratton, J. Fluoresc. 8, 365 (1998)

2. C. Dietrich, L. A. Bagatolli, Z. N. Volovyk, N. L. Thompson, M. Levi, K. Jacobson, E. Gratton, Biophys. J. 80, 1417 (2001)

3. Z. R. Grabowski, K. Rotkiewicz, W. Rettig, Chem. Rev. 103, 3899 (2003)

4. B. N. Davis, C. J. Abelt, J. Phys. Chem. A 109, 1295 (2005)

5. C. H. Kim, T. Joo, Phys. Chem. Chem. Phys. 11, 10266 (2009)

6. C. H. Kim, T. Joo, Opt. Express 16, 20742 (2008) 